\title{
Instrumentos de Avaliação da Imagem Corporal e dos Hábitos Alimentares na Anorexia Nervosa: Análise da Produção Científica
}

Instruments For The Assessment Of Body Image And Food Habits In Anorexia Nervosa: Analysis Of

Scientific Production

Instrumentos De Evaluación De La Imagen Corporal Y De Los Hábitos Alimenticios En La Anorexia Nerviosa: Análisis De La Producción Científica

Carolina Leonidas \&

Manoel Antônio dos

Santos

Universidade

de São Paulo
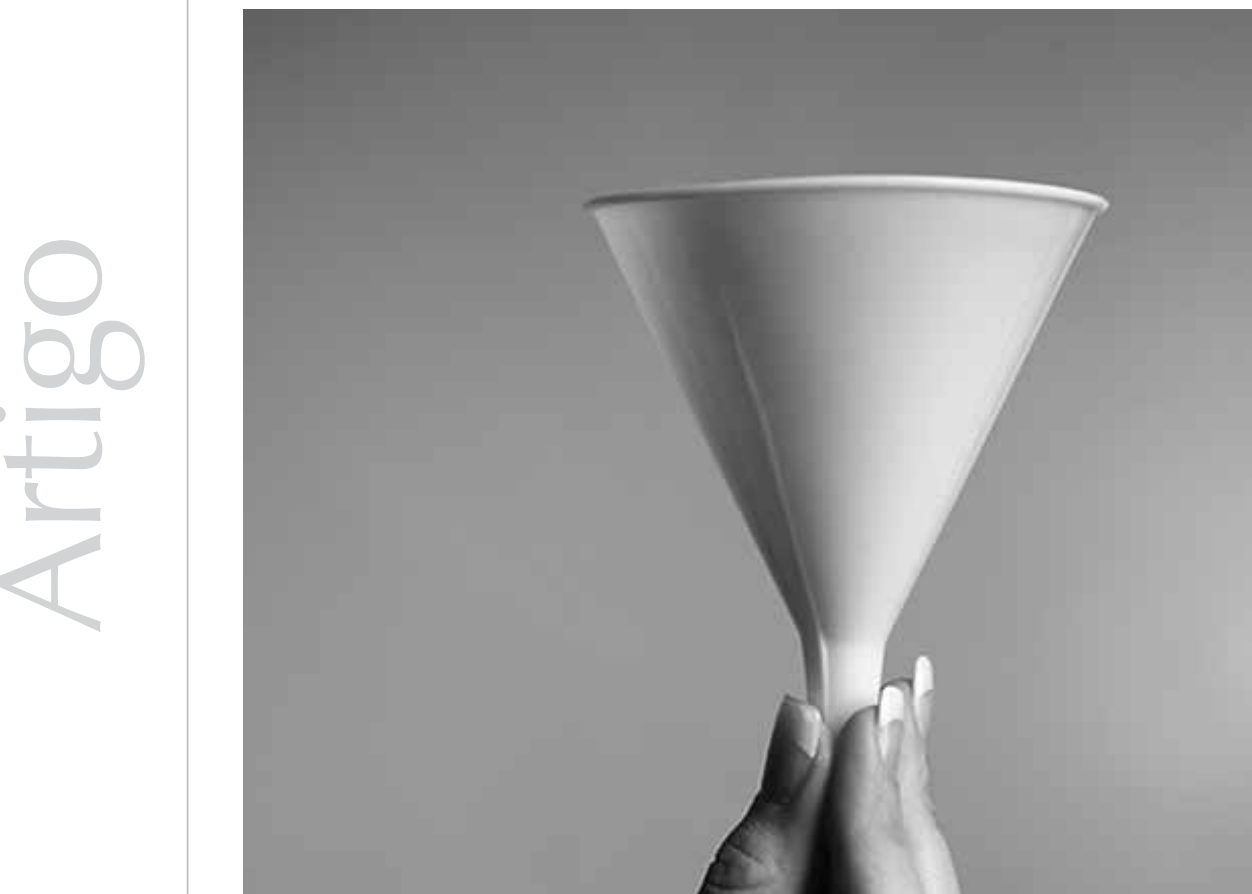
Resumo: Este estudo é uma revisão integrativa da literatura que tem o objetivo de descrever os instrumentos de mensuração utilizados na avaliação da imagem corporal e dos hábitos alimentares na anorexia nervosa. As bases consultadas foram LILACS e SciELO. Das 98 referências levantadas, oito foram selecionadas e recuperadas na íntegra para extração dos dados, constituindo o corpus da pesquisa. Constatou-se o predomínio de publicações na última década, principalmente a partir de 2000. Em termos gerais, os resultados indicaram que há poucos instrumentos que avaliam a imagem corporal e os hábitos alimentares de forma aprofundada, sendo que a maioria dos instrumentos encontrados visava a avaliar os sujeitos e a estabelecer o diagnóstico de transtorno alimentar (TA). Evidenciou-se, assim, a necessidade de mais estudos na área de elaboração e de validação de testes, escalas e inventários que tratem das síndromes parciais dos TAs, tais como a distorção da imagem corporal.

Palavras-chave: Anorexia nervosa. Imagem corporal. Distúrbios do ato de comer. Instrumentos de avaliação.

\begin{abstract}
The aim of this integrative review of the literature is to describe the instruments used to assess body image and food habits in nervous anorexia. The examined databases were LILACS and SciELO. Ninety-eight references were identified, eight of which were selected, and full articles were consulted for data extraction, constituting the corpus of the research. There was a predominance of publications in the last decade, mainly from 2000 on. Overall, results indicated that there are few instruments to assess body image and food habits in detail, and most of the instruments found were aimed at assessing the patients and diagnosing eating disorder (ED). This evidenced the need for further studies in the field of test, scale and inventory development and validation which address the partial syndromes of ED, such as body image distortion.
\end{abstract}

Keywords: Anorexia nervosa. Body image. Eating disorders. Assessment instruments.

Resumen: Esta investigación es una revisión integrativa de la literatura que tiene el objetivo de describir los instrumentos de mensuración utilizados en la evaluación de la imagen corporal y de los hábitos alimenticios en la anorexia nerviosa. Las bases consultadas fueron LILACS y SciELO. De las 98 referencias relevadas, ocho fueron seleccionadas y recobradas integralmente para extracción de los datos, constituyendo el corpus de la investigación. Se constató el predominio de publicaciones en la última década, principalmente desde el año 2000. En términos generales, los resultados indicaron que hay pocos instrumentos que evalúan la imagen corporal y los hábitos alimenticios de forma profundizada, siendo que la mayoría de los instrumentos encontrados visaba evaluar los sujetos y establecer el diagnóstico de trastorno alimenticio (TA). Se evidenció, así, la necesidad de más estudios en el área de elaboración y de validación de pruebas, escalas e inventarios que traten de los síndromes parciales de los TA, tales como la distorsión de la imagen corporal.

Palabras clave: Anorexia nerviosa. Imagen corporal. Transtorno de la ingestion de alimentos. Instrumentos de evaluación.

O DSM-IV-TR ${ }^{T M}$ - Manual Diagnóstico e Estatístico de Transtornos Mentais, 4 $\underline{\underline{a}}$ edição (Associação Americana de Psiquiatria, 2002) - classifica a anorexia nervosa (AN) como um tipo de transtorno alimentar (TA) que se caracteriza por recusa do indivíduo em manter um peso corporal na faixa normal mínima de acordo com sua idade e altura. Esse transtorno envolve temor intenso de ganhar peso e uma perturbação significativa na percepção da forma ou do tamanho do corpo, o que resulta em caquexia, ou seja, em fraqueza geral e má disposição corporal decorrente da grave desnutrição (Claudino \& Borges, 2002). A AN pode ser dividida em dois subtipos: o restritivo, caracterizado pela perda substancial de peso em curto intervalo de tempo devido a dietas, jejuns ou excesso de exercícios físicos, e o purgativo, no qual ocorre uma compulsão alimentar seguida de rituais compensatórios, como vômitos autoinduzidos ou uso abusivo de laxantes, diuréticos ou drogas anorexígenas.

A AN tem sua origem na complexa interação de vários fatores que se conjugam na produção dos sintomas. Dentre eles, podem ser destacados: vulnerabilidade genética, traços de personalidade, psicopatologia parental, experiências adversas e pressões socioculturais, entre outros (Morgan, Vecchiatti, \& Negrão, 2002). A literatura propõe a hipótese de influência combinada da dinâmica familiar (confusão das fronteiras que definem os subsistemas familiares, que dificultaria a formação da identidade e da individualidade nos membros da família), do meio cultural e de aspectos da personalidade 
do indivíduo como fatores concorrentes na etiologia dos distúrbios relacionados com a distorção da imagem corporal, que predispõem ao desenvolvimento dos TAs (Oliveira \& Santos, 2006).

A distorção da imagem corporal é uma característica psicopatológica importante da AN, considerada critério diagnóstico pelo DSM-IV-TR ${ }^{\mathrm{TM}}$ (Associação Americana de Psiquiatria, 2002). Essa perturbação no modo de vivenciar a forma corporal fomenta a busca desenfreada pela magreza e a negação dos riscos associados ao emagrecimento excessivo (Saikali, Soubhia, Scalfaro, \& Cordás, 2004; Peres \& Santos, 2006).

\section{Imagem corporal}

O corpo tem sido objeto de interesse de diversos campos do saber, e pode ser estudado a partir de vários ângulos: corpo biológico, corpo da anatomia e dos estudos intervencionistas da Medicina, corpo social, corpo em interação com outros corpos, corpo estético e da beleza corporal - que ganham cada vez mais espaço na mídia e no imaginário das pessoas - , corpo antropológico, corpo histórico e corpo subjetivo da psicanálise (Lazzarini \& Viana, 2006).

Para Mandet (1993), a psicanálise abarca o corpo como objeto para o psiquismo, ou seja, o corpo como representação inconsciente e que está investido em uma relação de significado, construído a partir das vivências do sujeito e da incorporação de dados do mundo, marcando e sendo marcado por ele. Desse modo, o corpo pode ser considerado uma fonte de experiências afetivas de prazer e dor, de preocupação e satisfação, de medo e desejo, e não apenas de um espaço físico que limita a individualidade em meio ao espaço social; é, portanto, uma superfície de contato na qual feixes de múltiplas sensações se desenvolvem mas que também envolve uma dimensão que transcende os limites da sensorialidade, já que é sede dos desejos e impulsos despertados pelo modo singular de o sujeito relacionar-se consigo mesmo e com os outros (Peres, Santos, \& Kruschewsky, 2007). Nesse sentido, o corpo é um conjunto sofisticado de zonas erógenas que adquirem significados emocionais que particularizam os seres humanos e que organizam sua subjetividade. Assim se define o construto da corporeidade.

Segundo Peres e Santos (2006), o contato com o seio materno, proporcionado pela amamentação, revela para a criança a existência de um mundo externo, fomentando os rudimentos da distinção entre interior e exterior. A partir dessa experiência primordial, a representação mental do corpo começa a formar-se, e tem origem o complexo processo de construção da imagem corporal. Esse processo ocorre incessantemente ao longo da vida, e é moldado pelas vivências afetivas do indivíduo, pelas restrições ou pela permissibilidade que encontra no ambiente em que se desenvolve e pelos recursos de seu aparelho psíquico (Peres, Santos, \& Kruschewsky, 2007).

Na concepção de Schilder (1994/1935), a imagem corporal é a representação mental que temos de nosso esquema corporal. Essa projeção do corpo como imagem mental organiza-se como um núcleo vital na constituição da personalidade, uma vez que corresponde a um conjunto funcional que permite que o indivíduo se diferencie do outro, estabelecendo as fronteiras de seu ego e as bases emocionais para seu desenvolvimento emocional. O modo singular como cada sujeito configura essa imagem, consciente ou inconscientemente, assume um significado primordial na manutenção da saúde e no desenvolvimento de sua corporeidade, motricidade e vitalidade, com reflexos na constituição da autoestima e nos relacionamentos afetivos e sociais. 
As práticas alimentares, de modo geral, são muito diversificadas e alteram-se com frequência, possivelmente como

decorrência dos modismos alimentares, do grande número de dietas e das novas práticas

para perder peso divulgadas diariamente pela mídia (Sicchieri, Bighetti, Borges, Dos Santos, \& Ribeiro, 2006).
Um componente importante nos TA e que tanto pode influenciar como ser influenciado pela imagem corporal é a autoestima (Shea \& Pritchard, 2007). A autoestima parece estar associada à manutenção da insatisfação corporal crônica e do desejo incoercível de emagrecer, e, quando associada a outras variáveis, pode acarretar a ocorrência do TA. Entre as variáveis influenciadoras do quadro psicopatológico, estão incluídos os hábitos alimentares.

\section{Hábitos alimentares}

Pode-se entender como prática ou comportamento alimentar todas as formas de convívio com o alimento, que têm início no momento da tomada de decisão de que alimento preparar, a forma de prepará-lo, os utensílios utilizados, as características, os horários e a divisão da alimentação em porções nas refeições do dia, as preferências e as aversões que se estabelecem, e que é encerrado com a digestão (Dunker \& Phillipi, 2006).

As práticas alimentares, de modo geral, são muito diversificadas e alteram-se com frequência, possivelmente como decorrência dos modismos alimentares, do grande número de dietas e das novas práticas para perder peso divulgadas diariamente pela mídia (Sicchieri, Bighetti, Borges, Dos Santos, \& Ribeiro, 2006). No entanto, sabe-se que as pessoas acometidas por $\mathrm{AN}$ - portanto, que restringem a ingesta alimentar - iniciam sua dieta com um jejum progressivo, abolindo os alimentos calóricos a princípio, seguindo-se a restrição de outros grupos de alimentos, exercendo elas, assim, um rígido controle sobre o comportamento alimentar (Sicchieri et al., 2006). Pode-se afirmar, portanto, que as práticas alimentares não se resumem apenas aos alimentos que são ingeridos ou deixados de ingerir, mas compreendem também as regras e os significados que permeiam todos os aspectos relativos à prática de consumo de alimentos (Alessi, 2006).
A forma como a distorção da imagem corporal se manifesta é de grande interesse, juntamente aos hábitos alimentares das pacientes com $\mathrm{AN}$ - que inclui tanto os alimentos que são evitados quanto a forma como elas se posicionam perante algumas situações que envolvem a alimentação - e os sentimentos que permeiam essas situações. Compreender a forma pela qual as pessoas com AN vivenciam sua imagem corporal pode subsidiar importantes recursos para a elaboração de estratégias de diagnóstico e para um tratamento psicológico mais efetivo, colaborando no estabelecimento do prognóstico não apenas em termos da elucidação dos aspectos psicológicos implicados mas também na recuperação do estado nutricional, já que, a partir do momento em que a paciente se sente satisfeita com seu corpo, suas atitudes alimentares podem, gradativamente, evoluir no sentido da aquisição de padrões saudáveis.

Nos limites desse campo de investigação, há uma série de estudos que destacam as escalas e os instrumentos utilizados para mensurar a imagem corporal das pessoas com AN, permitindo avaliar o grau de distorção da autoimagem que elas apresentam. São diversos os instrumentos empregados para avaliar os hábitos alimentares, ou seja, para caracterizar o padrão de comportamento alimentar, apontando eventuais restrições, purgações e minuciosidades na alimentação. A maior parte dos instrumentos foi desenvolvida e validada no âmbito internacional. Nessa vertente, é relevante inquirir sobre os possíveis estudos acerca do construto da imagem corporal e da avaliação dos hábitos alimentares de pessoas com AN no contexto brasileiro. Tendo em vista a necessidade de amadurecimento das pesquisas nessa área, pode-se indagar: quais seriam os instrumentos mais utilizados pelos autores nacionais? Seriam adaptações, com a necessária adaptação transcultural e validação daqueles utilizados nos países centrais, como Estados Unidos e países europeus? Ou seriam 
instrumentos produzidos especialmente para o cenário brasileiro? Quais os pressupostos teórico-metodológicos que sustentam essas técnicas de avaliação psicológica e como eles repercutem na ampliação do saber acerca da AN, notadamente no Brasil?

Considerando a relevância dessa questão de pesquisa, este estudo pretende contribuir para fortalecer a discussão sobre avaliação psicológica no contexto da AN a partir de uma revisão integrativa da literatura científica acerca dos instrumentos de mensuração utilizados no Brasil. Buscou-se evidenciar o número de artigos publicados em fontes de pesquisa de impacto no contexto brasileiro e o perfil dos estudos, de modo a identificar as tendências apontadas por essas publicações no que concerne aos instrumentos de mensuração e, assim, discutir possíveis lacunas e perspectivas de produção científica no Brasil, de modo a possibilitar maior direcionamento das pesquisas futuras sobre o tema.

\section{Método}

A revisão integrativa foi eleita como método de pesquisa, pois permite sumarizar estudos já finalizados acerca da temática abordada. Além disso, esse tipo de revisão, quando realizada de forma crítica, mantém os mesmos padrões de rigor, clareza e replicação das pesquisas primárias (Ganong, 1987). Conforme preconizado por essa autora, embora os métodos para a condução de revisões integrativas possam variar, há procedimentos a serem seguidos, como o estabelecimento de critérios para inclusão e exclusão dos estudos publicados e análise crítica dos seus resultados.

A partir dos procedimentos destacados pela literatura, na realização da presente revisão foram cumpridas as seguintes etapas: seleção da questão temática, estabelecimento dos critérios para a seleção da amostra, análise e interpretação dos resultados e apresentação da revisão (Leonidas \& Santos, 2012).

\section{Procedimento}

Para a realização da revisão integrativa, foram selecionados os seguintes descritores, segundo o DeCS - Descritores em Ciências da Saúde: anorexia nervosa, imagem corporal / body image e hábitos alimentares / food habits. Tendo em vista que o propósito era encontrar artigos que apresentassem uma intersecção entre os temas propostos, as buscas nas bases de dados foram realizadas utilizando-se os unitermos de maneira combinada. Para tanto, utilizou-se o operador booleano and com o objetivo de definir, para o sistema de busca, como deveria ser feita a combinação entre os unitermos. Dessa forma, as buscas foram realizadas por meio das seguintes palavras-chave: anorexia nervosa and imagem corporal, anorexia nervosa and hábitos alimentares, anorexia nervosa and body image, anorexia nervosa and food habits, imagem corporal and hábitos alimentares, body image and food habits.

Após o levantamento preliminar das publicações nas bases de dados escolhidas, os resumos foram lidos e analisados segundo os critérios de inclusão/exclusão preestabelecidos, que serão descritos na sequência. Os artigos selecionados tiveram seus resumos lidos minuciosamente. Em um segundo momento, foram recuperados os estudos na íntegra, que foram lidos e analisados utilizando-se um formulário elaborado pelos pesquisadores. Em seguida, os artigos foram submetidos ao crivo dos critérios de inclusão e de exclusão, constituindo-se assim o corpus de análise da revisão.

\section{Bases de dados consultadas}

Visando a assegurar uma abrangência dessa revisão no contexto brasileiro, foram 
consultadas as seguintes bases de dados: LILACS e SciELO Brasil.

\section{Período de publicação}

Foram selecionados artigos publicados entre os anos 1980 a 2012.

\section{Critérios de inclusão e exclusão dos artigos}

Foram definidos os seguintes critérios de inclusão: artigos empíricos, teóricos e de revisão acerca do tema (mensuração da imagem corporal e dos hábitos alimentares de pacientes com AN) que se referiam ao contexto brasileiro ou que tivessem sido produzidos no País.

Nesta revisão, foram excluídos trabalhos como teses, dissertações, livros, capítulos de livros, resenhas, cartas e editoriais. Essa escolha se deve ao fato de que, em uma das bases utilizadas (LILACS), notou-se a indexação dessas modalidades. São publicações que não foram submetidas a um processo de avaliação que envolve uma sistemática comparável ao peer review das revistas científicas. A revisão por pares é uma tradição em publicações científicas, e consiste em uma auditoria de qualidade realizada às cegas por outros pesquisadores com reconhecimento na área de conhecimento do manuscrito sob avaliação. Garante-se, assim, a qualidade do conhecimento veiculado pelo artigo e sua obediência aos padrões de rigor científico que devem nortear sua apreciação. A fim de realçar apenas os estudos submetidos a esse processo rigoroso de avaliação, optou-se por selecionar exclusivamente artigos indexados.

Também foram excluídos estudos a respeito de condutas alimentares de populações específicas - por exemplo, atletas, bailarinas, modelos e universitários, entre outros - e artigos que abordavam problemas de conduta alimentar secundários a transtornos orgânicos - por exemplo, obesidade, diabetes mellitus e hipertireoidismo, entre outros. Quadros de anorexia ou bulimia secundários a uma doença orgânica, ou condutas alimentares disfuncionais de populações específicas de risco não configuram um quadro psicopatológico de TA, pois não preenchem os critérios diagnósticos definidos pelo DSMIV-TR ${ }^{T M}$ - Manual Diagnóstico e Estatístico de Transtornos Mentais, 4르 edição (Associação Americana de Psiquiatria, 2002).

Outros eixos excluídos foram: AN sob a ótica da Sociologia, da psicanálise e da fenomenologia, que foram excluídos por se tratarem de trabalhos teóricos e/ou empíricos que tinham como objetivo realizar uma descrição da AN sem incluir instrumentos de avaliação da imagem corporal ou dos hábitos alimentares, que é o objetivo da presente revisão, quadro de obesidade, relação entre atividade física e TA, comportamentos alimentares de risco em adolescentes, perfeccionismo e TA e TA no atendimento de emergência (pronto-socorro). Foram excluídos, ainda: temas muito distantes daqueles abrangidos pela presente revisão - por exemplo, dismorfia muscular, abuso de álcool, estudos que focalizam outros TAs que não a $A N$, investigações a respeito dos efeitos de determinados medicamentos e relatos de experiência. Excluíram-se também estudos ligados exclusivamente à área médica (neurobiologia da imagem corporal, por exemplo) e também os artigos oriundos de outras áreas do saber que não apresentavam relação direta com a Psicologia ou com o enfoque desta investigação.

Artigos repetidos, isto é, que apareceram mais de uma vez, em diferentes combinações de palavras-chave ou nas bases bibliográficas consultadas, foram computados uma única vez. 


\section{Resultados}

Nas bases bibliográficas selecionadas, foram encontrados 98 referências (resumos) a partir das combinações dos unitermos de busca, sendo que 44 eram repetidos. Desses trabalhos, 82 foram encontrados na base LILACS e 16 foram recuperados na SciELO.

A partir da aplicação dos critérios de inclusão e de exclusão adotados, chegou-se a um total de oito artigos indexados, produzidos no contexto nacional, que abordavam instrumentos de mensuração e de investigação da imagem corporal e dos hábitos alimentares de pacientes com AN. Essa amostra reduzida pode ser considerada um reflexo da escassa produção literária brasileira acerca dos construtos abordados no presente estudo, o que sugere a necessidade de produção de mais estudos nessa área.

Os artigos selecionados foram recuperados e analisados na íntegra. De um modo geral, os artigos abordavam a distorção da imagem corporal e os hábitos alimentares disfuncionais como algumas das características típicas da AN, porém não se restringiam apenas à análise desses construtos, mas também de outros aspectos envolvidos no quadro. Assim, foram escassos os estudos que abordavam especialmente a questão da imagem corporal e dos hábitos alimentares, os quais foram integralmente selecionados para análise e discussão. Essas publicações encontram-se sumarizadas na Tabela 1.

Tabela 1. Instrumentos de mensuração mencionados e/ou investigados nos artigos selecionados

Autores Ano de publicação $\begin{aligned} & \text { Instrumentos de mensuração mencionados/ } \\ & \text { investigados }\end{aligned}$

Freitas, Gorestein \&

Appolinario

2002

Dunker \& Philippi 2003

Saikali, Soubhia, Scalfaro \& Cordás

2004

Moya et al.

2005

Sicchieri, Bighetti,

Borges, Santos \&

Ribeiro

2006
Revisão não sistemática da literatura acerca de vários instrumentos de medida, dentre eles: Body Shape Questionnaire (BSQ), Body Dissatisfaction Scale (BDS) of the Eating Disorders Scale (EDI), Three-Factor Eating Questionnaire (TFEQ) e Eating Inventory.

Recordatório alimentar de 24 horas, Eating Attitudes Test (EAT-26) e um questionário de comportamento alimentar, desenvolvido pelas próprias autoras, para verificar preferências e aversões alimentares.

Os autores mencionam o Body Shape Questionnaire (BSQ) como um dos principais instrumentos de avaliação da imagem corporal nos TA.

Seção de transtornos alimentares do Development and Well-Being Assessment (DAWBA).

Não há menção a instrumentos padronizados de mensuração, porém registra a importância do registro alimentar e do recordatório alimentar de 24 horas. 
continuação da tabela 1

\begin{tabular}{lll}
\hline Autores & Ano de publicação & Instrumentos de mensuração mencionados/investigados \\
\hline Peres \& Santos & Autores utilizam o desenho da figura humana (DFH), \\
& mas também mencionam outros instrumentos: Body \\
& Shape Questionnaire (BSQ), Body Dissatisfaction \\
& Scale (BDS), Body-Self Relations Questionnaire \\
& (BSRQ) e Body Image Avoidance Questionnaire \\
& (BIAQ) como os mais utilizados pelos pesquisadores \\
& na investigação da imagem corporal na AN.
\end{tabular}

Peres \& Santos $2011 \quad$ Revisão sistemática da literatura, com o objetivo de verificar a utilização de técnicas projetivas na avaliação de características psicopatológicas de pacientes com TA.

Oliveira-Cardoso \&

Santos

2012
Avaliação do funcionamento lógico, afetivo e adaptação social de pacientes com AN e BN por meio da aplicação do método de Rorschach, com análise de dados baseada nas normas da Escola Francesa.

Ressalta-se ainda que, apesar do aumento da prevalência de casos de AN no Brasil e no mundo (Paccola, 2006), e da distorção da imagem corporal ser, segundo o DSM-IV-TR ${ }^{\text {TM }}$ (Associação Americana de Psiquiatria, 2002), um dos principais critérios diagnósticos para identificar esses quadros, poucos são os estudos que abordam essa questão de forma específica. Essa constatação deve ser entendida como um convite para a realização de novas pesquisas, que possam ampliar a produção de conhecimento a respeito desse componente tão importante da AN.

No que concerne ao ano de publicação, apenas um artigo foi publicado na década de 1980, mais especificamente, em 1985. Essa publicação foi encontrada a partir da busca com as combinações: anorexia nervosa and imagem corporal e anorexia nervosa and body image. Os outros artigos foram publicados a partir de 1994, sendo a maioria deles entre os anos 2000 e 2008. Esse dado sugere que o pico de produção científica se deu na primeira década do novo milênio.

No que se refere ao perfil dos estudos selecionados e recuperados, todos apresentam uma breve contextualização dos TAs (incluindo a bulimia nervosa - BN), sendo que quatro deles dão maior ênfase à AN. Quatro desses estudos também utilizam (ou mencionam) instrumentos de avaliação quantitativa da imagem corporal (Body Shape Questionnaire - BSQ e Body Dissatisfaction Scale - BDS of the Eating Disorders Scale - EDI) e dos hábitos alimentares (Three-Factor Eating Questionnaire - TFEQ ou Eating Inventory, e o Eating Attitudes Test - EAT-26). Um deles apresenta a validação da Seção de Transtornos Alimentares do Development and Well-Being Assesment (DAWBA), que é um pacote de questionários, entrevistas e técnicas de avaliação desenvolvido para gerar diagnósticos baseados no DSM-IV e CID-10 de anorexia nervosa, bulimia nervosa e respectivas síndromes parciais, em estudos epidemiológicos com jovens de 7 a 17 anos.

A seguir, considerando o objetivo deste estudo, serão apresentados e discutidos de modo 
pormenorizado os estudos que focalizam os instrumentos de mensuração da imagem corporal e dos hábitos alimentares na $\mathrm{AN}$, publicados e/ou desenvolvidos no contexto brasileiro de produção.

\section{Discussão}

Em um primeiro estudo recuperado, Peres e Santos (2006) afirmam que os instrumentos padronizados possibilitam a expressão de sentimentos e de comportamentos tidos como vergonhosos - tais como a ojeriza à gordura e a utilização de métodos purgativos - que poderiam ser mascarados em uma entrevista clínica e que, por isso, se mostram de especial relevância para a adequada compreensão dos casos. No entanto, os autores também pontuam que esses instrumentos apresentam limitações, já que o construto da imagem corporal pressupõe elementos inconscientes que os instrumentos quantitativos não conseguem acessar. Assim, os autores propõem que a utilização de técnicas projetivas no estudo da imagem corporal pode favorecer a apreensão dos elementos inconscientes. Por esse motivo, delinearam um estudo utilizando o desenho da figura humana (DFH), com o objetivo de avaliar a imagem corporal de mulheres com AN, buscando investigar suas dimensões inconscientes. Trata-se de uma técnica projetiva gráfica que consiste em solicitar ao examinando que faça o desenho de uma figura humana e, em seguida, o desenho de uma figura humana do sexo oposto ao da primeira. Os materiais empregados para tanto são um lápis preto e folhas de papel em branco tamanho ofício. Após a coleta dos desenhos, um inquérito específico é utilizado com o intuito de complementar a atividade gráfica com a comunicação verbal.

O estudo de Peres e Santos mostrou que a imagem corporal de pacientes diagnosticadas com AN apresenta marcante distorção decorrente de sentimentos de inferioridade desencadeados por uma preocupação patológica com a própria forma física. Além disso, mostrou também que as mulheres em questão apresentam acentuada fragilidade egóica, são propensas à utilização de mecanismos de defesa arcaicos, caracterizamse por uma restrição do potencial adaptativo, controlam os próprios impulsos com excessivo rigor e tendem à passividade, à introversão, à obsessividade e à dependência afetiva. Esses resultados são consistentes com os achados da literatura científica especializada, o que indica que o DFH se mostrou adequado frente ao objetivo proposto, pois, segundo Peres e Santos, possibilitou a projeção de "conteúdos internos situados para além do plano da consciência, que exercem papel determinante na constituição da imagem corporal e que somente poderiam ser apreendidos mediante a utilização de métodos mais dispendiosos" (2006, p. 367).

No entanto, os autores pontuam que a utilização do DFH apresenta certas limitações, já que as características de uma produção gráfica não necessariamente revelam aspectos da imagem corporal ou da personalidade do indivíduo que a executou, visto que podem ser influenciadas por diversas variáveis, tais como determinantes sociais e culturais, fatores de gênero e condições ambientais. Por isso, a adequada avaliação dos desenhos encontra-se intimamente relacionada à experiência do examinador no manejo do instrumento, o que sem dúvida restringe suas possibilidades de utilização em larga escala. Além disso, no cenário brasileiro, a utilização de técnicas de avaliação psicológica com finalidade diagnóstica é prerrogativa exclusiva do psicólogo, o que inviabiliza seu emprego pelos demais profissionais de saúde que integram as equipes multidisciplinares.

Saikali, Soubhia, Scalfaro e Cordás (2004) abordam a questão da imagem corporal de forma diferenciada. Esses autores observam que a perturbação da imagem corporal inclui 
Saikali et al. ressaltam ainda que a distorção da imagem corporal pode estar

presente em

outros quadros psicopatológicos, tais como: transtorno dismórfico

corporal, delírios somáticos,

transtorno da identidade de gênero (transexualismo), depressão, esquizofrenia e obesidade,

porém, segundo esses autores, é nos TA que seu papel

sintomatológico e prognóstico se mostra mais proeminente. diversos distúrbios, dentre eles: consciência cognitiva do próprio corpo, consciência das sensações corporais, senso de controle sobre as funções corporais e razões afetivas para a realidade da configuração corporal. Além disso, para os mencionados autores, a imagem corporal também é permeada pelos mecanismos: pensamento dicotômico, comparação injusta, atenção seletiva e erro cognitivo.

Os estudos de Saikali et al. (2004) e de Peres e Santos são convergentes no que diz respeito ao pressuposto de que, na $\mathrm{AN}$, mais alarmante do que a má nutrição em si é sua associação com distorção severa da imagem corporal que, dentre outros modos de expressão, apresenta-se como uma ausência de preocupação com a magreza extrema. Saikali et al. ressaltam ainda que a distorção da imagem corporal pode estar presente em outros quadros psicopatológicos, tais como: transtorno dismórfico corporal, delírios somáticos, transtorno da identidade de gênero (transexualismo), depressão, esquizofrenia e obesidade, porém, segundo esses autores, é nos TA que seu papel sintomatológico e prognóstico se mostra mais proeminente.

Freitas, Gorestein e Appolinario (2002) realizaram um estudo que descreve os principais instrumentos e métodos utilizados para mensurar os aspectos psicopatológicos gerais e específicos dos TAs, dentre eles a imagem corporal e o comportamento alimentar que, na maioria das vezes, contribuem para a manutenção do transtorno e dificultam seu tratamento. Esses autores destacam que os TAs têm etiologia multifatorial, e, por esse motivo, são necessárias avaliações que abordem os vários componentes envolvidos em sua gênese e manutenção. Assim, a aplicação de instrumentos que avaliam as comorbidades - especialmente os sintomas depressivos e ansiosos -, a imagem corporal, a qualidade de vida e a adequação social, além daqueles utilizados para o rastreamento ou diagnóstico do transtorno em si, são de grande utilidade para melhor compreensão do quadro clínico, bem como para a elaboração de estratégias de intervenção mais adequadas.

Alguns dos instrumentos autoaplicáveis mais utilizados para avaliação dos quadros de TAs, segundo Freitas, Gorestein e Appolinario (2002), são:

- Eating Attitudes Test (EAT-26), que se apresenta em duas versões: uma com 40 itens, e outra menor, com 26 itens, esta última validada no Brasil por Bighetti, Santos, Dos Santos e Ribeiro (2004). Esse teste é utilizado para o rastreamento de indivíduos suscetíveis ao desenvolvimento de $\mathrm{AN}$ ou $\mathrm{BN}$, e indica a presença de padrões alimentares anormais, mas não revela a possível psicopatologia subjacente.

- Eating Disorder Inventory (EDI), composto por 64 itens que avaliam as características psicológicas e comportamentais comuns à AN e à $\mathrm{BN}$, e consiste de oito subescalas, três das quais avaliam os aspectos psicopatológicos específicos dos TAs (ímpeto para a magreza, insatisfação corporal e bulimia). As outras cinco avaliam os aspectos psicopatológicos gerais observados em pacientes com TAs. $\mathrm{O}$ EDI apresenta uma versão revisada, o EDI210, que, além dos itens da escala original, contém mais três subescalas (asceticismo, regulação do impulso e insegurança social), totalizando 91 itens. Há uma versão do EDI para crianças (KEDS). O teste pode ser utilizado para o rastreamento de indivíduos com alto risco de desenvolverem um TA. Em pesquisa clínica, pode ser útil como medida de desfecho e como indicador prognóstico em estudos de tratamento.

- Eating Disorder Examination versão questionário (EDE-Q), constituído por 41 itens. Seu resultado é apresentado no formato de quatro subescalas: restrição alimentar, preocupação alimentar, preocupação 
com a forma corporal e preocupação com o peso. As subescalas mostram-se altamente correlacionadas, e sua validade e confiabilidade estão bem documentadas. É considerado um instrumento útil para o rastreamento dos TAs.

Os instrumentos padronizados utilizados para mensurar especificamente os quadros de $\mathrm{BN}$ e os do transtorno de compulsão alimentar periódica (TCAP) não serão apresentados no presente estudo pelo fato de este ser restrito apenas à descrição dos aspectos concernentes aos quadros de AN.

Outro instrumento desenvolvido para gerar diagnósticos baseados nos critérios diagnósticos definidos pelo DSM-IV e CID-10 de $\mathrm{AN}$, de $\mathrm{BN}$ e das respectivas síndromes parciais em estudos epidemiológicos é a Seção de Transtornos Alimentares do Development and Well-Being Assessment (DAWBA), desenvolvida e validada no Brasil por Moya et al. (2005). Essa seção do teste é composta por 53 perguntas, sendo que 42 são estruturadas e 11, semiestruturadas. Esses itens englobam questões a respeito da distorção da imagem corporal, peso e altura, insight, medo de ganhar peso, desejo por alimentos, presença e frequência de purgações, comportamentos para perda de peso, comportamentos compensatórios, sintomas menstruais, consequências físicas de comportamentos alimentares anormais e o impacto de sintomas relevantes no bemestar e no funcionamento social de pessoas jovens. Cada questão - ou grupo de questões - corresponde aos critérios diagnósticos do DSM-IV ou da CID-10 para TAs.

O estudo de validação do teste mostrou que, para a detecção de qualquer TAs segundo o DSM-IV e a CID-10, o diagnóstico final apresentou $100 \%$ de sensibilidade, $94 \%$ de especificidade, $88 \%$ de valor preditivo positivo, $100 \%$ de valor preditivo negativo e $95 \%$ de concordância teste-reteste.
Assim, Moya et al. afirmam que a Seção de Transtornos Alimentares do DAWBA apresenta boas propriedades psicométricas, que a qualificam para uso em estudos clínicos e epidemiológicos.

Para a avaliação da imagem corporal especificamente, Freitas et al. (2002) e Saikali et al. (2004) mencionam o Body Shape Questionnaire (BSQ) como o instrumento de mensuração mais utilizado. Esse questionário mede o grau de distorção da imagem corporal por meio da pontuação total. Trata-se de uma escala do tipo Likert de seis pontos, e constitui-se de 34 perguntas autopreenchíveis para serem respondidas segundo a legenda: nunca (1 ponto), raramente (2 pontos), às vezes ( 3 pontos), frequentemente (4 pontos), muito frequentemente (5 pontos) e sempre (6 pontos). As pontuações podem indicar diferentes níveis de distorção da imagem corporal: sem distorção, distorção leve, distorção moderada e distorção grave.

Freitas et al. também mencionam o Body Dissatisfaction Scale(BDS) of the Eating Disorders Inventory (EDI) como importante instrumento de avaliação da imagem corporal. Trata-se de uma subescala do EDI que avalia crenças relacionadas a partes específicas do corpo, tais como a convicção de que as coxas, o quadril ou as nádegas são muito grandes. Esse instrumento tem mostrado correlação estatisticamente significativa com outras medidas de transtorno da imagem corporal.

Em relação à avaliação da personalidade, Peres e Santos (2011) realizaram uma revisão focalizando as técnicas projetivas utilizadas na avaliação de aspectos psicopatológicos da AN e BN. As principais características psicopatológicas reportadas foram: passividade, introversão e obsessividade, conflitos, tensões e angústias em relação à figura materna, grave distorção da imagem corporal e tendência à fusão nos relacionamentos interpessoais. Os autores constataram escassez 
de pesquisas nacionais com utilização de técnicas projetivas junto a pacientes com TAs. As análises dos dados obtidos evidenciaram predomínio de metodologias incipientes em termos de sofisticação das evidências proporcionadas pelos resultados reportados, uma vez que foram adotadas amostras clínicas reduzidas e selecionadas com base em critérios diagnósticos que não são claramente reconhecidos. Nesse sentido, os autores enfatizam a necessidade de novas pesquisas que envolvam o emprego de técnicas projetivas no contexto dos TAs, para que os profissionais da área tenham subsídio de evidências empíricas que possam ser aproveitadas nas avaliações multidisciplinares da população brasileira.

Um dos artigos selecionados na presente revisão (Oliveira-Cardoso \& Santos, 2012) utilizou o método de Rorschach no contexto da avaliação da personalidade de 27 mulheres com AN e/ou BN. Os autores avaliaram o funcionamento lógico, o afetivo e a adaptação social dessas mulheres, utilizando-se as normas da Escola Francesa para codificação e interpretação dos dados. Evidenciou-se que as participantes apresentavam capacidade produtiva e desejo de estabelecerem relacionamentos afetivos. No entanto, eram facilmente invadidas por afetos não elaborados, que predispunham ao isolamento social e à vivência de inquietações e de ansiedade que, por sua vez, eram escoadas pela via somática. Também foi evidenciado o comprometimento das funções psíquicas das participantes, indicando a necessidade de acompanhamento psicoterapêutico integrado à equipe multiprofissional. Nesse sentido, o método de Rorschach mostrou-se um instrumento adequado para a avaliação da dinâmica psíquica de pacientes com TA. No que diz respeito aos hábitos alimentares, o instrumento de avaliação mais utilizado, segundo Freitas et al., é o Three-Factor Eating Questionnaire (TFEQ), instrumento autopreenchível, com propriedades psicométricas estabelecidas, também denominado Eating Inventory. O questionário contém três subescalas, que avaliam três dimensões do comportamento alimentar: restrição cognitiva, desinibição e fome percebida. Esses aspectos são importantes para melhor entendimento do distúrbio do comportamento alimentar associado aos TA e à obesidade, e sua utilização, na prática clínica, auxilia a definição da intervenção terapêutica mais apropriada a cada paciente.

Os procedimentos que Freitas et al. (2004) denominam "instrumentos de automonitoramento" também são bastante utilizados. Consistem em diários alimentares nos quais os pacientes anotam a ingestão alimentar diária (hora do dia e local, quantidade e qualidade dos alimentos sólidos e líquidos ingeridos), geralmente no período de uma semana. É também solicitado ao paciente que registre seus afetos, cognições e mecanismos compensatórios relacionados à alimentação. Sicchieri et al. (2006), em estudo que descreve o manejo e os cuidados nutricionais prestados pela equipe do Grupo de Assistência em Transtornos Alimentares (GRATA), do Hospital das Clínicas da Faculdade de Medicina de Ribeirão Preto, relatam que os instrumentos mais utilizados para avaliação dos comportamentos alimentares das pacientes são o registro alimentar e o recordatório alimentar de 24 horas, ou seja, instrumentos de automonitoramento. Esses recursos possibilitam observar características e descrever ações específicas de cada paciente, ilustradas pelo comportamento alimentar, tais como: alimentação infantilizada (composta por mingaus, sopas e papinhas), crença de que podem ganhar peso se utilizarem insumos de higiene pessoal ("pasta dental engorda", "condicionador de cabelos contém calorias") ou se consumirem determinados alimentos sólidos ou líquidos ("água cria barriga") e utilização de medicação fitoterápica com 
efeito laxativo/diurético após as refeições (Sicchieri et al., 2006).

Tais procedimentos também podem ser utilizados para avaliar os alimentos consumidos por uma população específica, como no estudo de Dunker e Philippi (2003). Esses autores utilizaram o recordatório alimentar de 24 horas para avaliar os alimentos consumidos por estudantes adolescentes que apresentavam sintomas anoréxicos, identificados por meio da aplicação do Eating Attitudes Test (EAT-26). Os alimentos mais consumidos foram: frutas, hortaliças, leite desnatado e bala, e os menos consumidos foram: refrigerante, chocolate, massas e batata frita. Os alimentos de que "mais gostam" foram, em ordem decrescente: massas, carnes, hortaliças e doces, e os de que "menos gostam" foram: hortaliças, carnes e gorduras. Houve aversão maior por doces nas adolescentes com sintomas de AN. Esse estudo possibilita visualizar o alto número de alunas com sintomas e que apresentam aversões alimentares semelhantes às das pacientes com AN, o que justifica a implementação de programas de intervenção nutricional visando à prevenção de TAs.

Apesar de esses instrumentos oferecerem uma riqueza de detalhes e de informações sobre os sintomas dos transtornos relativos aos hábitos e aos comportamentos alimentares do indivíduo, Sicchieri et al. (2006) pontuam que eles também apresentam algumas limitações: superestimativa das quantidades ingeridas, omissão ou manipulação de informações importantes (como número de purgações diárias, de refeições realizadas, ou mesmo de negação da fome), além do aspecto crítico relacionado à veracidade e à confiabilidade das informações como um todo.

\section{Considerações finais}

Concluindo o percurso desta revisão integrativa, nota-se que houve, nas últimas décadas, uma predominância de publicações a respeito das síndromes parciais envolvidas nos quadros de TAs, principalmente a distorção da imagem corporal e a aquisição de hábitos alimentares disfuncionais. No entanto, a maioria dos estudos disponíveis no âmbito da AN relaciona esse transtorno a outros quadros psicopatológicos - como, por exemplo, à depressão - e a amostras específicas de segmentos sociais mais vulneráveis, como bailarinas, atletas, adolescentes e estudantes, entre outros.

A partir dos resultados obtidos, constatouse que não foi localizado nenhum estudo com instrumentos de mensuração da imagem corporal ou dos hábitos alimentares de pacientes com AN que tenham sido desenvolvidos originalmente no Brasil. Além disso, há diversos instrumentos largamente utilizados nos países centrais e da Europa que não foram validados em nosso país.

A revisão integrativa também possibilitou notar que não há grande variedade de instrumentos que avaliem a imagem corporal e os hábitos alimentares especificamente. A maioria dos testes utilizados no Brasil não aborda a imagem corporal como objeto principal de avaliação, mas como componente que é parte de um construto maior - ou seja, o transtorno alimentar, a personalidade do indivíduo avaliado, entre outros. Por outro lado, grande parte dos instrumentos utilizados para avaliação e estabelecimento de diagnóstico de TAs engloba perguntas ou grupos de perguntas que investigam o construto da imagem corporal e os comportamentos alimentares adotados pelo sujeito, uma vez que a caracterização desses componentes é crucial 
para o estabelecimento do diagnóstico de TAs de acordo com os critérios propostos pelo DSM-IV ou pela CID-10. Vale ressaltar que a imagem corporal corresponde a uma dimensão subjetiva da personalidade, e, como tal, não pode ser apreendida de forma completa e totalizante por testes e escalas. No entanto, aventa-se a hipótese de que, se os testes tivessem como objetivo avaliar o construto da imagem corporal de forma predominante - e não apenas como parte constituinte do TAs -, seria possível obter uma ideia mais apurada da forma como o indivíduo percebe seu corpo e as emoções relacionadas a essa percepção.

Também é válido ressaltar que a presente revisão apresenta limitações, uma vez que não foram incluídos estudos realizados em outros países além do Brasil. Considera-se de suma importância a realização de pesquisas futuras que abordem os instrumentos de avaliação da imagem corporal e dos hábitos alimentares utilizados em outros países, possibilitando que os pesquisadores brasileiros entrem em contato com novos instrumentos, aprimorando suas técnicas de avaliação e de intervenção.
Nesse panorama, destacamos a necessidade de futuras investigações sobre o assunto, com o objetivo de fornecer maiores esclarecimentos a respeito da questão da imagem corporal e dos hábitos alimentares em pacientes com AN, já que a melhora da distorção da imagem do corpo pode favorecer a aquisição de hábitos alimentares saudáveis e a redução dos rituais purgativos. Acredita-se que o aumento do número de publicações nessa área pode proporcionar maior compreensão entre os profissionais, possibilitando que se conheçam as reais implicações das síndromes parciais de TAs sobre a saúde e a qualidade de vida do indivíduo acometido, assim como da importância de a imagem corporal e os hábitos alimentares serem constantemente avaliados e examinados com o paciente pelos membros da equipe multidisciplinar, de forma a promover melhora global do quadro psicopatológico. Assim, considera-se que novas publicações acerca dessa temática poderão fornecer evidências seguras para orientar a prática clínica. 


\section{Carolina Leonidas}

Doutoranda pelo Programa de Pós-graduação em Psicologia da Faculdade de Filosofia, Ciências e Letras de Ribeirão Preto da Universidade de São Paulo - FFCLRP-USP. Membro do Laboratório de Ensino e Pesquisa em Psicologia da Saúde - LEPPS (USP - CNPq), Ribeirão Preto - SP - Brasil.

Email: cleonidas@usp.br

\section{Manoel Antônio dos Santos}

Doutor em Psicologia Clínica pela Universidade de São Paulo e livre-docente pela Faculdade de Filosofia, Ciências e Letras de Ribeirão Preto da Universidade de São Paulo - FFCLRP-USP. Coordenador do Laboratório de Ensino e Pesquisa em Psicologia da Saúde - LEPPS (USP - CNPq), Ribeirão Preto - SP - Brasil.

Email: masantos@ffclrp.usp.br

Endereço para envio de correspondência:

Departamento de Psicologia. Laboratório de Ensino e Pesquisa em Psicologia da Saúde - LEPPS (FFCLRP-USP-CNPq). Avenida Bandeirantes, 3900, Monte Alegre. CEP:

14040-901. Ribeirão Preto, SP.

Recebido 10/09/2012, 1aㅡ Reformulação 05/02/2013, Aprovado 11/04/2013. 
Alessi, N. P. (2006). Conduta alimentar e sociedade. Medicina, Ribeirão Preto, 39(3), 327-332.

Associação Americana de Psiquiatria. (2002). DSM-IV-TR ${ }^{T M}$ Manual Diagnóstico e Estatístico de Transtornos Alimentares: texto revisado (C. Dornelles, trad., 4a. ed. rev.). Porto Alegre: Artmed.

Bighetti, F., Santos, C. B. Dos Santos, J. E. \& Ribeiro, R. P. P. (2004). Tradução e validação do Eating Attitudes Test (EAT-26) em adolescentes do sexo feminino na cidade de Ribeirão Preto-SP. Jornal Brasileiro de Psiquiatria, 53(6), 339-346.

Claudino, A. M., \& Borges, M. B. F. (2002). Critérios diagnósticos para os transtornos alimentares: conceitos em evolução. Revista Brasileira de Psiquiatria, 24(3), 7-12. doi: http:// dx.doi.org/10.1590/S1516-44462002000700003

Dunker, K. L., \& Phillipi, S. T. (2003). Hábitos e comportamentos alimentares de adolescentes com sintomas de anorexia nervosa. Revista de Nutrição, 16(1), 51-60. doi: http://dx.doi. org/10.1590/S1415-52732003000100006

Freitas, S., Gorestein, C., \& Appolinario, J. C. (2002). Instrumentos para a avaliação dos transtornos alimentares. Revista Brasileira de Psiquiatria, 24(3), 34-38. doi: http:// dx.doi.org/10.1590/S1516-44462002000700008

Ganong, L. H. (1987). Integrative reviews of nursing research. Research in Nursing \& Health, 10, 1-11. doi: 10.1002/ nur.4770100103

Lazzarini, E. R., \& Viana, T. C. (2006). O corpo em psicanálise. Psicologia: Teoria e Pesquisa, 22(2), 241-249. doi: http:// dx.doi.org/10.1590/S0102-37722006000200014

Leonidas, C., \& Santos, M. A. (2012). Imagem corporal e hábitos alimentares na anorexia nervosa: uma revisão integrativa da literatura. Psicologia: Reflexão e Crítica, 25(3), 550-558. doi: http://dx.doi.org/10.1590/S0102-79722012000300015

Mandet, E. S. C. (1993). La fascinación de los significados: Una problemática acerca de la noción de cuerpo en psicoanalisis. Psicoanalisis con Niños e Adolescentes, 4, 114-124.

Morgan, C. M., Vecchiatti, I. R., \& Negrão, A. B. (2002). Etiologia dos transtornos alimentares: aspectos biológicos, psicológicos e sócio-culturais. Revista Brasileira de Psiquiatria, 24(3), 18-23. doi: http://dx.doi.org/10.1590/S151644462002000700005
Moya, T., Bacy, F. B., Goodman, R., Nogueira, F. C., Focchi, P. S., Nicoletti, M. et al. (2005). The Eating Disorders Section of the Development and Well-Being Assessment (DAWBA): development and validation. Revista Brasileira de Psiquiatria, 27(1), 25-31. doi: http://dx.doi.org/10.1590/S151644462005000100008

Oliveira, E. A., \& Santos, M. A. (2006). Perfil psicológico de pacientes com anorexia e bulimia nervosas: a ótica do psicodiagnóstico. Medicina, Ribeirão Preto, 39(3), 353-360.

Oliveira-Cardoso, E. A., \& Santos, M. A. (2012). Avaliação psicológica de pacientes com anorexia e bulimia nervosas: indicadores do método de Rorschach. Fractal: Revista de Psicologia (online), 24(1), 159-174. doi: http://dx.doi. org/10.1590/S1984-02922012000100011

Paccola, A. T. F. (2006). Escuta do psiquiatra: sinais e sintomas de anorexia nervosa e bulimia nervosa. Medicina, Ribeirão Preto, 39(3), 349-352.

Peres, R. S., \& Santos, M. A. (2006). Contribuições do desenho da figura humana para a avaliação da imagem corporal na anorexia nervosa. Medicina, Ribeirão Preto, 39(3), 361-370.

Peres, R. S., \& Santos, M. A. (2011). Técnicas projetivas na avaliação de aspectos psicopatológicos da anorexia e bulimia. PsicoUSF, 16(2), 185-192. doi: http://dx.doi.org/10.1590/ S1413-82712011000200007

Peres, R. S., Santos, M. A., \& Kruschewsky, M. S. L. B. (2007). Imagem corporal em obesos mórbidos antes e depois de uma intervenção multidisciplinar. Psychologica, 44, 467-88.

Saikali, C. J., Soubhia, C. S., Scalfaro, B. M., \& Cordás, T. A. (2004). Imagem corporal nos transtornos alimentares. Revista de Psiquiatria Clínica, 31(4), 164-166.

Schilder, P. (1994). A imagem do corpo: as energias construtivas da psique (R. Wertman, trad.). São Paulo: Martins Fontes (Trabalho original publicado em 1935).

Shea, M. E., \& Pritchard, M. E. (2007). Is self-esteem the primary predictor of disordered eating? Personality and Individual Differences, 42(8), 1527-1537. doi: ttp://dx.doi. org/10.1016/j.paid.2006.10.026

Sicchieri, J. M. F., Bighetti, F., Borges, N. J. B. G., Dos Santos, J. E., \& Ribeiro, R. P. P. (2006). Manejo nutricional nos transtornos alimentares. Medicina, Ribeirão Preto, 39(3), 371-374. 